\title{
Fixation of Distal Tibial Fractures by Intramedullary Nail with Multidirectional Distal Locking Screws
}

\author{
MOHAMED E. TAHA, M.Sc.; ALI M. EMRAN, M.D.; KAMAL M. HAHEZ, M.D. and \\ NABIL O. GHARBO, M.D.
}

The Department of Orthopaedic Surgery, Faculty of Medicine, Tanta University

\begin{abstract}
Background: Fractures of the distal tibia involve the diaphyseo-metaphyseal area of the bone, and may be either extra-articular or intrarticular. Several methods have been described for the treatment of these fractures, including casting, external and internal fixation.
\end{abstract}

Aim of Study: The aim of the work was to evaluate the results of fixation of distal tibial fractures by intramedullary nail with multidirectional distal locking screws (Expert tibial nail).

Methods: This study included 21 patients with distal tibial fractures treated between October 2017 and October 2018 (including follow-up period) at Tanta University Hospital with intramedullary nail with multidirectional distal locking screws (Expert tibial nail). Clinical and radiological data were analyzed.

Results: According to Tenny \& Wiss scoring systems for distal tibial fractures the clinical results were graded as excellent in six patients $(28.6 \%)$, good in nine patients $(38.1 \%)$, fair in four patients $(19 \%) \&$ poor in two patients $(9.5 \%)$. The excellent and good results were considered as satisfactory results while the unsatisfactory included the fair and the poor results. Thus, satisfactory results were found in 15 patients $(71.43 \%)$, and the unsatisfactory results were found in six patients $(28.57 \%)$.

Conclusion: Treatment of distal tibial fractures using intramedullary nail with multidirectional distal locking screws (Expert nail) is a safe and accepted method alternative to conventional nail and plating technique. It provides additional biomechanical stability than the conventional nails and plate osteosynthesis.

Key Words: Distal tibial fractures - Intramedullary nail Multidirectional distal locking screws.

\section{Introduction}

FRACTURES of the distal tibia involve the diaphyseo-metaphyseal area of the bone, and may be either extra-articular or intrarticular [1]. They are

Correspondence to: Dr. Mohamed E. Taha, The Department of Orthopaedic Surgery, Faculty of Medicine, Tanta University one of the most challenging injuries in orthopedic trauma because of the high risk of complications which may result from the nature of the injury itself, secondary to surgery, or both [2].

They account for less than $10 \%$ of all fractures of the lower extremity, and occur more frequently in men than women. Although these fractures occur in all age groups, they are rare in children and elderly. These fractures occur most often following a high energy trauma usually secondary to a motor vehicle accident $[3]$. Several methods have been described for the treatment of these fractures, including casting, external and internal fixation [4].

In the past, plate fixation achieved an acceptable degree of reduction and rigid fixation. The technique was used widely, but it required relatively extensive wound exposure and soft-tissue dissection. These factors increase the risk of infection and nonunion, especially for fractures with a severe soft-tissue injury or open fractures. In recent years, closed reduction and minimally invasive plating have provided superior options for treating these fractures. At the same time, locked intramedullary nailing has been widely accepted as a satisfactory treatment of tibial fracture. However, in patients with distal tibial fractures, there is a risk that the fracture will propagate into the ankle or that the nail will fail because of inadequate fixation of the small distal fragment [5]

The construction of intramedullary nails has improved greatly. The indications for their use have been extended to fractures closer to distal segments [6].

The Expert tibial nail is a new kind of multidirectional locked tibial intramedullary nail. Its interlocking system was developed to attain in- 
creased angular stability and to enhance the axial and lateral stability of fracture fragments. Because of the increased stability attained by the nail fixing of proximal or distal tibial fractures, the indications for use of the nail were expanded. The nail currently represents an effective approach to the treatment of complex tibial fractures such as distal tibial metadiaphyseal fractures [7].

This new multidirectional locked intramedullary system involves multiple locking options in different planes at the proximal and distal ends. The system has an angular stability locking system that enhances the axial and lateral stability of the fracture fragments [8]. Because of this modified design, the new system has advantages over the traditional tibial intramedullary nail in dealing with both proximal and distal tibial fractures. In addition, there are four advanced distal locking options in this new tibial intramedullary nail. The set-up involved one oblique locking option placed very distally, which allowed for optimized bone purchase and prevented damage to the soft tissue, as well as two mediolateral locking options and one anteroposterior locking option for better stabilization of the distal fragment. These design modifications ensure that angular stability is retained and anglestable locking reflects a potential to maintain fixation stability of distal tibia fractures [9]. All of these design modifications allow for better fixation of metaphyseal tibial segments through multiple interlocking holes in close proximity to either end of the nail.

The use of multidirectional interlocking screws ensures that alignment can be maintained and that stability can be preserved despite a short proximal or distal tibial segment [10].

The aim of the work was to evaluate the results of fixation of distal tibial fractures by intramedullary nail with multidirectional distal locking screws (Expert tibial nail).

\section{Patients and Methods}

\section{A- Patients:}

This study included 21 patients (16 males and 5 females) with distal tibial fractures treated between October 2017 and October 2018 (including follow-up period) at Tanta University Hospital with intramedullary nail with multidirectional distal locking screws (Expert tibial nail). Their ages ranged from 20 years to 59 years with an average of 36.38 years. The period of follow-up was up to six months.
Clinical examination:

\section{General examination:}

Blood pressure, Pulse, Temperature, Respiration other body systems.

Examination of the spine, knee, pelvis and calcaneus is very important for associated injury.

\section{Local examination:}

A- Inspection: Deformity, skin condition and presence of wound or associated injuries.

B-Palpation: Skin temperature, dorsalis pedis and posterior tibial pulse.

The inclusion criteria were Patients above the age of 18, (Type A) extra- articular metaphysical fractures according to the AO classification of fractures of distal tibia. With recent fractures within 14 days after the onest of trauma. Open fractures grade 1.

\section{B- Methods:}

All patients were examined both clinically \&laboratory to be sure that they were fit for operation. Preoperative antibiotics were used to prevent the risk of postoperative wound infection. IV antibiotics were given $30 \mathrm{~min}$. to $1 \mathrm{~h}$. before surgery. Spinal or general anaethesia were used in all patients. The patient was positioned supine on a radiolucent table with the ability to flex the knee by at least $90^{\circ}$. Position of the image intensifier was important such that visualisation of the tibia including the articular surface proximally and distally was possible in AP and lateral views. Incision was made in line with the central axis of the intra-medullary canal, this incision was transpatellar. The incision started proximally at the distal third of the patella along the patellar tendon down to the tibial tuberosity Fig. (1). The entry point was determined. It was giuded by the image intensifier. In the A.P. view the entry point was in line with the axis of the intramedullary canal and with the lateral tubercle of the intercondylar eminence. In lateral view the entry point was at the ventral edge of the tibial plateau Fig. (2).After determination of the entry point the awel was inserted into this point under contol of the image intensifier in A.P and lateral view.

The guide wire was inserted in central postion in AP and Lat. views and fracture was reduced by axi traction under the the image intensifier. In some cases the reduction was temporarily fixed by pecutanous reduction clamps. Then the intramedullary canal was reamed by flexible or rigid reamers over the guide wire to adjust the the diameter of the nail then the length of the nail was measured under 
the control the the image intensifier. The nail was inserted with the knee in at least $90^{\circ}$ flexion using the insertion handle with slight rotational movements. The passage of the nail through the fracture site was closely monitored by the the image intensifier. The correct proximal and distal positions of the nail were confirmed by the image intensifier in A.P. and lateral views Fig. (3). Consideration was given if primary compression or secondary dynamization was planned.

After confirmation of the nail postion, the guide wire was removed and the distal locking screws were inserted. Four screws were, two medial to lateral screws and two antro-posterior screws. In some cases three screws were sufficient to stabilize the distal fragment Fig. (4).

After insertion of distal locking screws and confirmation of reduction of distal fragment, two proximal locking screws were inserted.

The end cap was inserted to prevent ingrowth of soft tissue and bone into the nail. Reduction was assessed repeatedly during the different steps of the operation using both visual and image intensifier control. Angulation, length of both tibia and fibula, and rotation need to be considered, as well as the integrity of the ankle mortise. The aim is to achieve less than 5 degrees of varus or valgus, less than 5 degrees of anterior or posterior angulation. Most distal tibial fractures are accompanied by fibular fractures the fibular incision was placed slightly posterior to the mid coronal plan.

Plain X-ray was done (A.P. and lateral views) after the operation for the assessment of fracture reduction.

Immediate postoperative elevation of the limb for at least 2 or 3 days, active and passive range of motion of knee were started if the fibular fracture wasn't fixed.

Patients were discharged with instructions to start knee exercise with possibility for touch weight bearing for 6 weeks. In cases with fixed fibular fractures a below knee non-weight bearing cast was done for 6 weeks before beginning range of motion activities.

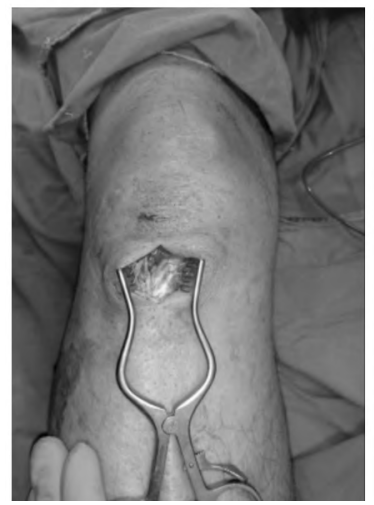

Fig. (1): Transpatellar incision.

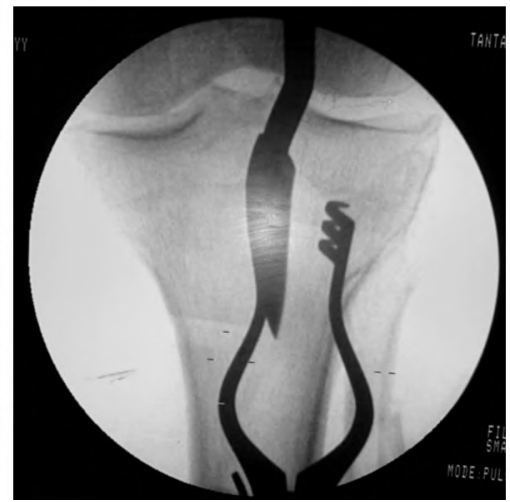

Fig. (2): Insertion of the awel.

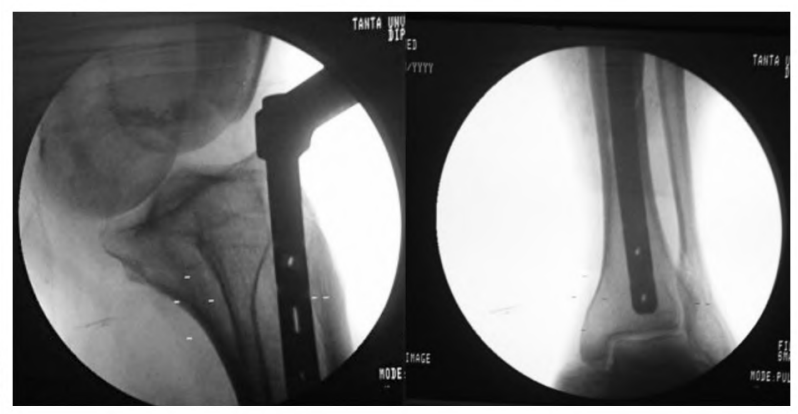

Fig. (3): Intaoperative position of nail proximally and distally.
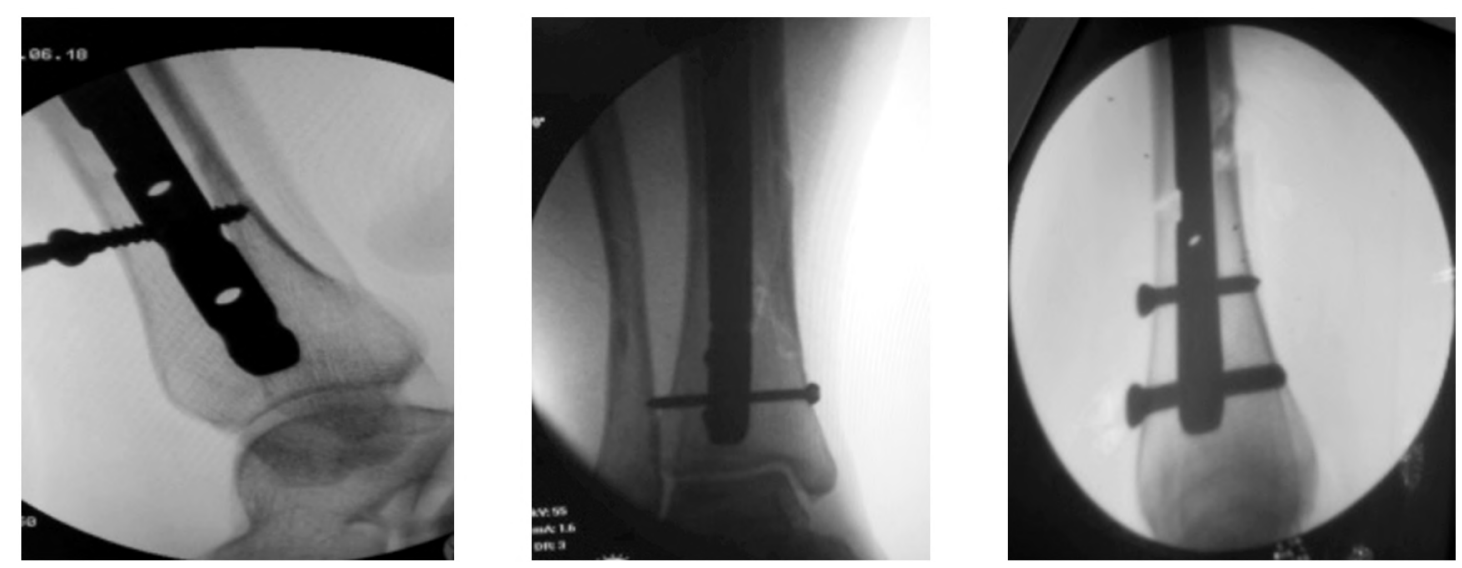

Fig. (4): Distal locking screws. 


\section{Results}

Follow-up period ranged from 3 to 6 months with a mean of 4.5 months.

\section{A- Clinical (functional) results:}

According to Tenny \& Wiss scoring systems for distal tibial fractures the clinical results were graded as excellent in six patients $(28.6 \%)$, good in nine patients $(38.1 \%)$, fair in four patients $(19 \%) \&$ poor in two patients $(9.5 \%)$. The excellent and good results were considered as satisfactory results while the unsatisfactory included the fair and the poor results. Thus, satisfactory results were found in 15 patients $(71.43 \%)$, and the unsatisfactory results were found in six patients (28.57\%) Fig. (5).

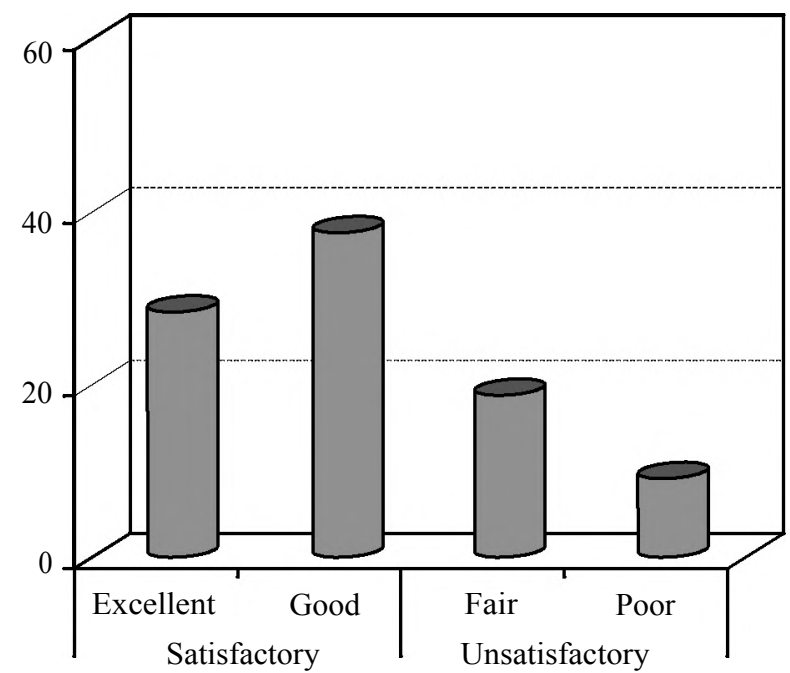

Fig. (5): Satisfactory and unsatisfactory results.

\section{Factors affecting the final clinical score:}

There was no significant correlation between the final end results and all the factors including (age, sex, and mode of trauma, associated fibular fractures, associated medical conditions, smoking and time lag).

\section{B- Radiological results:}

Radiographic follow-up of the patients assessed for maintenance of reduction, limb alignment, reestablishment of bone continuity, loosening of metal and callus formation.

\section{Time of union:}

This is the time in which the above mentioned criteria in both anteroposterior and lateral views of X-ray and also after the patient had no tenderness at fracture site. The time of union ranged from 12 to 24 weeks with mean of 16.23 weeks.
Factors affecting the time of union:

1- Age:

Comparing the time of union and age, significant correlation was found. $p$. value $=0.008$. Highest incidence of rapid union occurred in the age of 20-30 years Table (1).

Table (1): Relation between age and the time of union.

\begin{tabular}{lccc}
\hline Age group & $<16$ weeks & $>16$ weeks & Total \\
\hline $20-30$ & 8 & 0 & 8 \\
$>30-40$ & 2 & 4 & 6 \\
$>40-50$ & 3 & 1 & 4 \\
$>50-60$ & 1 & 2 & 3 \\
\hline Total & 14 & 7 & 21 \\
$p$. value & & 0.008 & \\
\hline
\end{tabular}

\section{2- Associated medical conditions:}

There was significant correlation between time of union and associated medical conditions (diabetes). $p$. value $=0.0502$.

Highest incidence of rapid union occured in non-diabetic patient (Table 2).

Table (2): Relation between associated medical condition and the time of union.

\begin{tabular}{lccc}
\hline $\begin{array}{l}\text { Medical conditions } \\
\text { (diabetes) }\end{array}$ & $<16$ weeks & $>16$ weeks & Total \\
\hline $\begin{array}{l}\text { Diabetic } \\
\text { Non diabetic }\end{array}$ & 1 & 3 & 4 \\
\hline Total \\
$p$.value & 13 & 4 & 17 \\
\hline
\end{tabular}

There was no significant correlation between the time of union and other factors including (sex, and mode of trauma, associated fibular fractures, smoking and time lag).

Complications:

Nonunion:

One case developed nonunion and further intervention had been done (dynamization and bone graft). It represented $4.76 \%$ of all cases.

\section{Delayed union:}

Three cases developed delayed union. Union started to appear after 20 weeks and completed at 24 weeks. It represented $14.28 \%$ of all cases.

\section{Sudeck's atrophy:}

Three cases developed Sudeck's atrophy. One case was 35 years old smoker male. And two case 
were diabetic one patient was 45 years old female and the other was 55 years old female. It represented $14.28 \%$ of all cases.

\section{Malunion:}

One case developed malunion due to malaligment after fixation. The case developed valgus ankle about 7 degrees. It represented $4.76 \%$ of all cases.

\section{Anterior knee pain:}

Four cases developed anterior knee pain. It was mainly due to the incision scar and infrapatellar pad scar. There was no need for continous medical treatment. It represented $19.04 \%$ of all cases.

\section{Cases:}

A 53 years old male patient, involved in motor vehicle accident caused right distal tibial fracture. $\mathrm{He}$ had associated proximal fibular fracture. He was operated one day after trauma. The fracture united after 12 weeks. According to Tenny \& Wiss scoring system, he had excellent functional score.
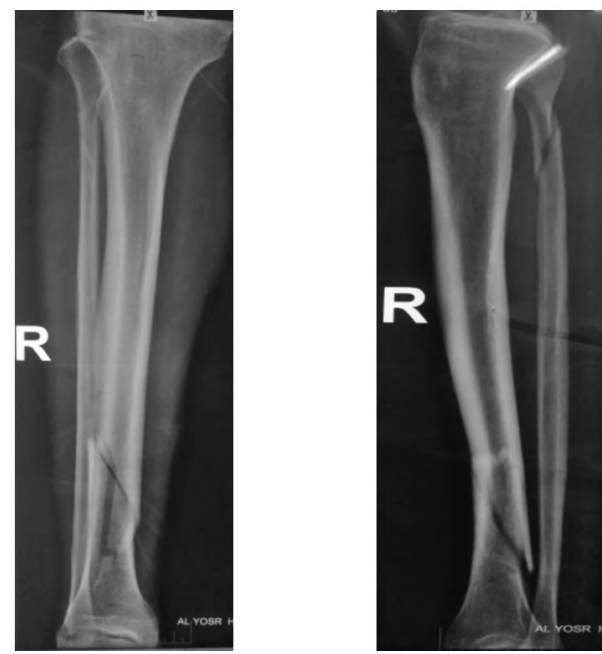

Fig. (6): Pre-operative X-rays; AP and Lateral views.
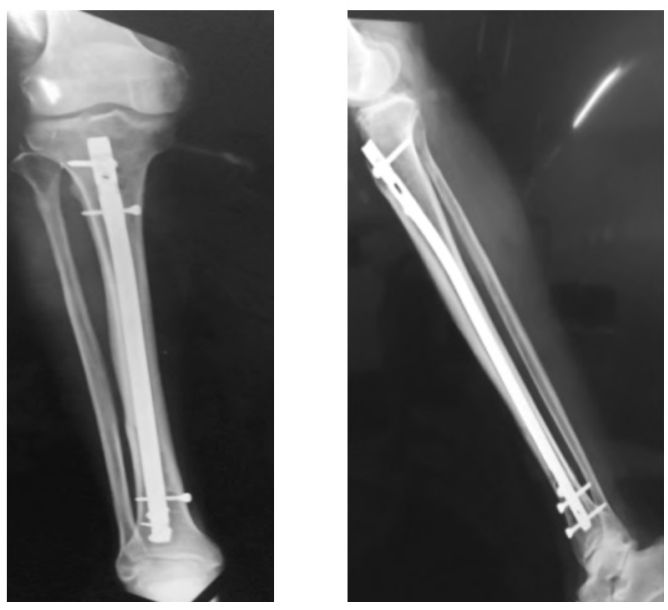

Fig. (7): Follow-up X-ray AP and lateral views after 3 months.
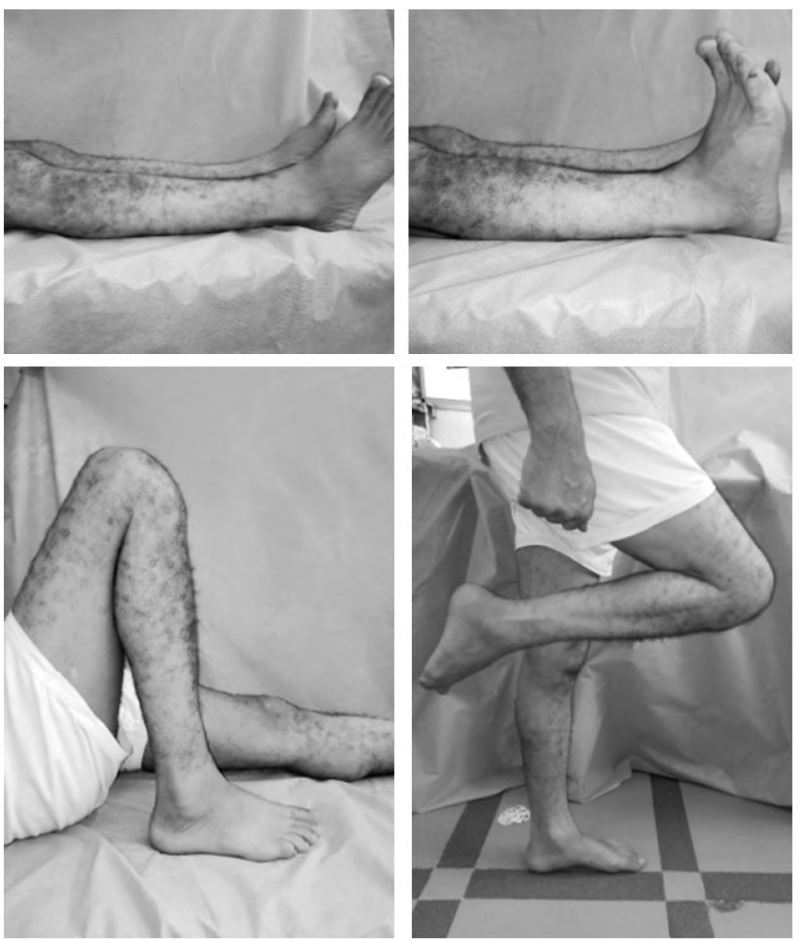

Fig. (8): Full range of motions after 3 months.
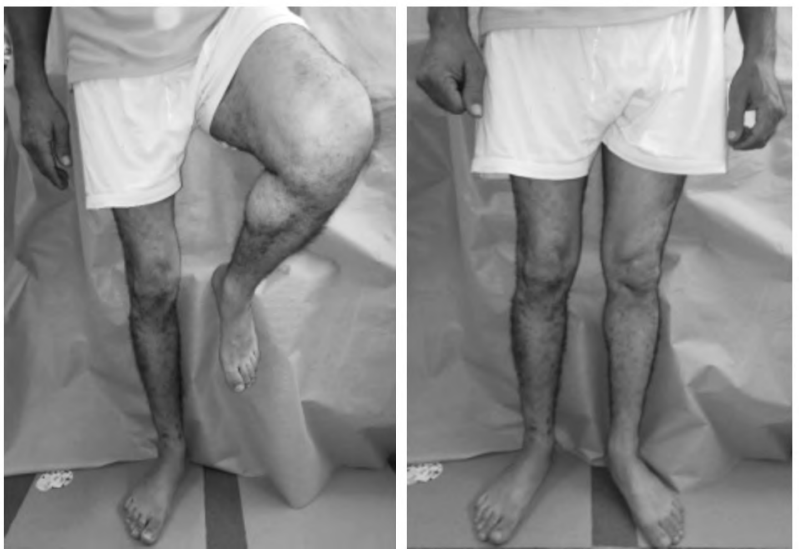

Fig. (9): Full weight bearing after 3 months.

\section{Discussion}

The optimal treatment of unstable distal tibial fracture remains controversial, despite the variety of treatment options which have been suggested for these injuries, including nonoperative treatment, plate fixation, intramedullary nailing, and external fixation. However, each of these treatment options has certain defects. The objectives in the treatment in these fractures are rapid and ideal healing, minimization of loss of function and prevention of any deformity [11].

Any method of treatment must satisfy certain criteria. Treatment should be simple, inexpensive, available in every centre dealing with trauma, minimally invasive to protect the vascularity of 
distal tibia with solid fixation and should be effective [12].

Compared to the study was done by Nadeem Ali et al. [13] sixty patients with extra articular distal tibial fractures were divided into 2 groups, the first group included 30 patients treated by distal tibial locking plating group (MIPO) and the second group included 30 patients treated by closed intramedullary interlocking nailing. In the first group, 21 patients $(70 \%)$ had satisfactory results, 8 patients (26.6\%) had fair results and one patient had poor results while in the second group, 19 patients $(63.3 \%)$ had satisfactory results, 9 patients $(30 \%)$ had fair results and 2 patients had poor results.

Compared to the study was done by Kartavya Chaudhari et al. [14] thirty patients were treated by LCP by MIPO, $63 \%$ had excellent results, $26 \%$ had good, $7 \%$ had fair and $4 \%$ had poor results. Twenty six patients were treated by Expert tibial nail, $54 \%$ had excellent results, $27 \%$ had good, $11 \%$ had fair and $8 \%$ had poor results.

Age ranged from 20 years to 59 years with an average of 36.38 years. Age was found to have statistically insignificant effect in the end results of this study but had statistically significant effect in the time of union. The highest incidence of satisfactory results occurred at the age of 20-30 years old. These results were nearly the same with that reported by Mohit Bihani et al. [15] in his study of intramedullary fixation of distal tibial fractures around diametaphysis using Expert tibial nail, who found that age had significant effect in the end results.

The time of union ranged from 12 to 24 weeks with mean of 16.23 weeks. This matched with the study was done by Neetin P. Mahajan et al. [16] 46 patients with distal tibial metadiaphyseal fractures were treated by Expert nail tibia, The average time of union was 16.4 weeks (ranged from 12 to 28 weeks). It also matched with the study was done by Ramachandra et al. [17] as the average time of union was 16 weeks of 72 patients with distal tibial fractures treated by expert nail tibia. Similar results reported by Kumar YC et al. [18] as the average time of union was 16 weeks for nailing (Expert nail) group and for plating group it was 18 weeks. However slower rates of union was reported by Mohit Bihani et al. [15] as the average time of union was 21.04 weeks.

In comparison with MIPPO, Guo et al. [19] reported no significant differences between nailing and MIPPO in terms of time to union, but nailing showed lesser operation and fluoroscopy use times, and better function and alignment. Other authors (92) preferred MIPPO because of the difficulty in controlling the distal fracture fragment with nailing because of the metaphyseal flare, wound complications because of poorer soft-tissue coverage, and the proximity to the ankle joint, which may amplify the bending moment of the short distal segment and may allow fracture propagation into the ankle joint.

In this study, three cases developed delayed union (14.28\%), three cases developed Sudeck's atrophy (14.28\%), one case developed malunion $(4.76 \%)$, one case developed nonunion $(4.76 \%)$ and anterior knee pain was found in four cases $(19.04 \%)$. So anterior knee pain represented the most common complication in this study.

Mohit Bihani et al. [15] in their study, also reported that commonest complication was anterior knee pain $(42 \%)$.

Markmiller et al. [20] in their study of fractures of the distal tibia treated with conventional intramedullary nail, reported that nearly half of his study population had a malalignment at the fracture site.

Compared to the study was done by Nadeem Ali et al. [13] malunion was the most common complication. Valgus malunion was the most type of malunion observed.

Compared to the study was done by Kartavya Chaudhari et al. [14] superficial skin infection $(10 \%)$ and ankle movement restriction (10\%) were the most common complications among patients treated by MIPO while anterior knee pain (3 8\%) was the most common complication among patients treated by Expert tibial nail.

The mean operation time was 150 (90-240) minutes. It was long duration Compared to Kumar YC et al. [18] who reported that the average duration of surgery was 88 minutes.

The relatively low number of patients, short follow-up period and the absence of control group are limitations to this study.

In general, this study emphasized the clinical success and low morbidity associated with the use of intramedullary nail tibia with multidirecrtional distal locking screws (expert nail). This was suited to the management of distal tibial fractures. Decreased incidence of soft tissue complications, early weight bearing and good functional recovery, all of these compare favorably with other reported treatments for patients with distal tibial fractures 
and substantiate the recommendations for expert nail should be the treatment of choice for such injuries.

\section{References}

1- HAHN M.P. and THIES J.W.: Pilon tibiale fractures. Chirurg, 75: 211-30, 2004.

2- ASSAL M., RAY A. and STERN R.: The extensile approach for the operative treatment of high-energy pilon fractures: Surgical technique and soft-tissue healing. J. Orthop. Trauma, 21: 198-206, 2007.

3- MARSH J.L., DIRSCHL D.R. and WEIGEL D.P.: Tibial plafond fractures. How do these ankles function over time? J. Bone Joint Surg. Am., 85: 287-95, 2003.

4- FISHER W.D. and HAMBLEN D.L.: Problems and pitfalls of compression fixation of long bone fractures: A review of results and complications. Injury, 10: 99-107, 1978.

5- TRAFTON P.G.: Tibial shaft fracture. Skeletal trauma. Philadelphia: W.B. Saunders, 1771-871, 1992.

6- NORK S.E., BAREI D.P., SCHILDHAUER T.A., et al.: Intramedullary nailing of proximal quarter tibial fractures. J. Orthop. Trauma, 20: 523-8, 2006.

7- NORK S.E., SCHWARTZ A.K., AGEL J., et al.: Intramedullary nailing of distal metaphysealtibial fractures. J. Bone Joint Surg. Am., 87: 1213-221, 2005.

8- GORCZYCA J.T., McKALE J., PUGH K., et al.: Modified tibial nails for treating distal tibia fractures. J. Orthop. Trauma, 16: 18-22, 2002.

9- GUEORGUIEV B., OCKERT B., SCHWIEGER K. , WAHNERT D., LAWSON-SMITH M., WINDOLF M. and STOFFEL K.: Angular stability potentially permits fewer locking screws compared with conventional locking in intramedullary nailed distal tibia fractures: A biomechanical study. J. Orthop. Trauma, 25: 340-6, 2011.

10- NEWMAN S.D., MAUFFREY C.P. and KRIKLER S.: Distal metadiaphysealtibial fractures. Injury, $41: 693$ 702, 2010.

11-REDFERN D.J., SYED S.U. and DAVIES S.J.: Fractures of the distal tibia, minimally invasive plate osteosynthesis. Injury, 35: 615-20, 2004.
12- COLES C.P. and GROSS M.: Closed tibial shaft fractures, management, treatment and complications a review of the prospective literature .Can. J. Surg., 43: 226-56, 2000.

13- NADEEM ALI, ABEDULLAH BHAT, FIRDOUS AHMED BANGROO, KHALID MUZZAFAR, SUHAIL AHMED BHAT, MANJEET SINGH DHANDA and MEHREEN MAQSOOD: Treatment of extra articular distal tibial fractures: Minimally invasive percutaneous plate osteosynthesis versus intramedullary nailing. Trauma Mon. July, 22 (4): e19593, 2017.

14- KARTAVYA CHAUDHARI, et al.: A comparative study of surgical management of distal tibial fractures treated by locking compression plate by minimally invasive percutaneous plate osteosynthesis (MIPPO) and expert tibial nail. EC Orthopedics, 9.9: 702-14, 2018.

15- MOHIT BIHANI, KRISHNA SRAVANTH P, SHARDAINDU SHARMA, RAJNEESH SOOD and FAHAD B.H.: Intramedullary fixation of distal tibial fractures around diametaphysis using locked intramedullary cannulated distal tibial nail aprospective study.International Journal of Orthopedics Sciences, 2: 38-42, 2016.

16- MAHAJAN N.P., PAWAR E.D., SUPE A.C. and MANAGUKIYA H.J.: Role of tibia interlocking nail in treating distal tibial metadiaphyseal fractures: study of 46 cases. International Journal of Research in Orthopedics, 4: 65$71,2018$.

17- RAMACHANDRA N. BADAMI1, SHANTHARAM SHETTY M. and AJITH KUMAR M.: Management of distal tibial metaphyseal fractures by expert tibial nail Indian Journal of Orthopaedics Surgery, 3 (3): 229-33, 2017.

18- YASHAVANTHA KUMAR C., SHIVAPRASED M.S and TRILOK V.: treatment of distal tibial fractures: Plating versus intramedullary nailing. International Journal of Research in Orthopedics, 2: 116-9, 2016.

19- GUO J.J., TANG N., YANG H.L. and TANG T.S. : A prospective, randomised trial comparing closed intramedullary nailing with percutaneous plating in the treatment of distal metaphyseal fractures of the tibia. J. Bone Joint Surg. Br., 92: 984-8, 2010.

20- MARKMILLER M., TJARKSEN M., MAYER E. and RUTER A.: The undreamed tibia nail multicenter study of the AO/ASIF. Langenbecks Arch. Surg., 385: 276-83, 2000 


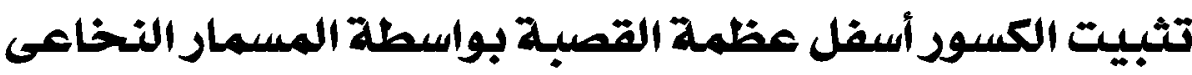

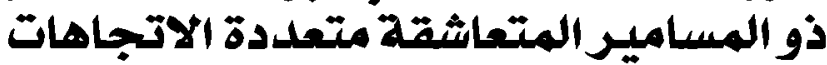

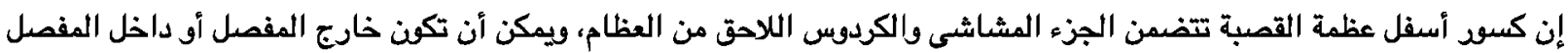

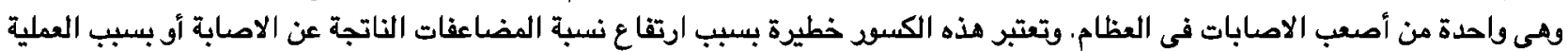
الجراحية أو كيهما.

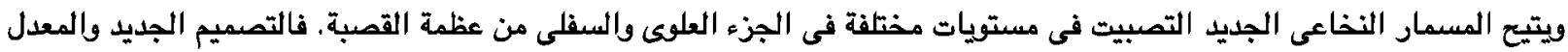

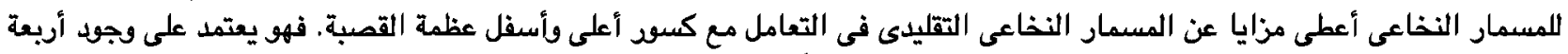

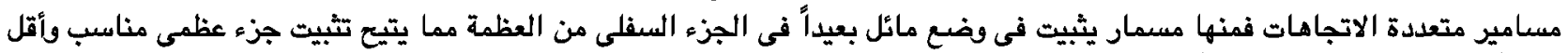

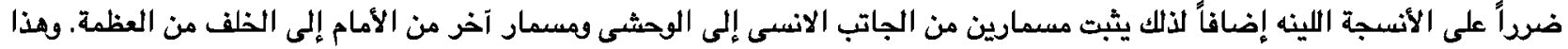

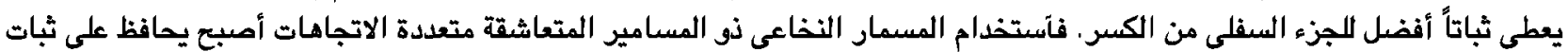

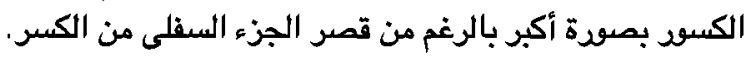

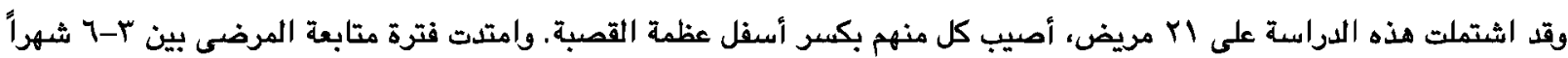

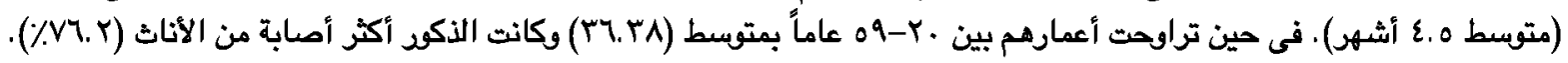

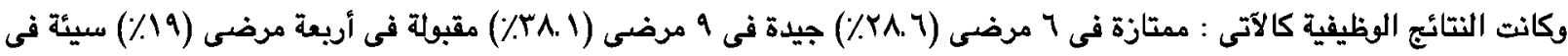

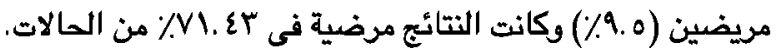

